\title{
Longitudinal analysis of cerebral aqueduct flow measures: multiple sclerosis flow changes driven by brain atrophy
}

\author{
Dejan Jakimovski ${ }^{1}$, Robert Zivadinov ${ }^{1,2}$, Bianca Weinstock-Guttman ${ }^{3}$, Niels Bergsland ${ }^{1,4}$, Michael G. Dwyer ${ }^{1}$ \\ and Marcella Maria Lagana ${ }^{4^{*}}$
}

\begin{abstract}
Background: Several small cross-sectional studies have investigated cerebrospinal fluid (CSF) flow dynamics in multiple sclerosis (MS) patients and have reported mixed results. Currently, there are no longitudinal studies that investigate CSF dynamics in MS patients.

Objective: To determine longitudinal changes in CSF dynamics measured at the level of aqueduct of Sylvius (AoS) in MS patients and matched healthy controls (HCs).

Materials and methods: Forty (40) MS patients and $20 \mathrm{HCs}$ underwent 3T MRI cine phase contrast imaging with velocity-encoded pulse-gated sequence at baseline and 5-year follow-up. For atrophy determination, MS patients underwent additional high-resolution 3D T1-weighted imaging. Measures of AoS cross-sectional area (CSA), average systolic and diastolic velocity peaks, maximal systolic and diastolic velocity peaks and average CSF flow rates were determined. Brain atrophy and ventricular CSF (VCSF) expansion rates were determined. Cross-sectional and longitudinal changes were derived by analysis of covariance (ANCOVA) and paired repeated tests. Confirmatory general linear models were also performed. False discovery rate (FDR)-corrected p-values lower than 0.05 were considered significant.

Results: The MS population demonstrated significant increase in maximal diastolic peak (from 7.23 to $7.86 \mathrm{~cm} / \mathrm{s}$, non-adjusted $p=0.037)$, diastolic peak flow rate $(7.76 \mathrm{ml} / \mathrm{min}$ to $9.33 \mathrm{ml} / \mathrm{min}$, non-adjusted $p=0.023)$ and AoS CSA (from 3.12 to $3.69 \mathrm{~mm}^{2}$, adjusted $p=0.001$ ). The only differentiator between MS patients and HCs was the greater AoS CSA (3.58 mm² vs. $2.57 \mathrm{~mm}^{2}$, age- and sex-adjusted ANCOVA, $p=0.045$ ). The AoS CSA change was associated with VCSF expansion rate (age- and sex-adjusted Spearman's correlation $r=0.496, p=0.019$ ) and not with baseline nor change in maximal velocity. The expansion rate of the VCSF space explained an additional $23.8 \%$ of variance in change of AoS CSA variance when compared to age and sex alone $\left(R^{2}=0.273, t=2.557\right.$, standardized $\beta=0.51$, and $\mathrm{p}=0.019$ ).
\end{abstract}

Conclusion: MS patients present with significant longitudinal AoS enlargement, potentially due to regional atrophy changes and ex-vacuo expansion of the aqueduct.

Keywords: Cerebrospinal fluid, Aqueduct of Sylvius, Brain atrophy, Multiple sclerosis, Phase contrast MRI

*Correspondence: mlagana@dongnocchi.it

${ }^{4}$ MRI Laboratory, CADiTeR, IRCCS, Fondazione Don Carlo Gnocchi ONLUS, Via Alfonso Capecelatro 66, 20148 Milan, Italy

Full list of author information is available at the end of the article

\section{Introduction}

Multiple sclerosis (MS) is chronic inflammatory and neurodegenerative disease of the central nervous system (CNS) characterized with intermittent events of neurological worsening followed by full or partial recovery. In

c) The Author(s) 2020. This article is licensed under a Creative Commons Attribution 4.0 International License, which permits use, sharing, adaptation, distribution and reproduction in any medium or format, as long as you give appropriate credit to the original author(s) and the source, provide a link to the Creative Commons licence, and indicate if changes were made. The images or other third party material in this article are included in the article's Creative Commons licence, unless indicated otherwise in a credit line to the material. If material is not included in the article's Creative Commons licence and your intended use is not permitted by statutory regulation or exceeds the permitted use, you will need to obtain permission directly from the copyright holder. To view a copy of this licence, visit http://creativeco mmons.org/licenses/by/4.0/. The Creative Commons Public Domain Dedication waiver (http://creativecommons.org/publicdomain/ zero/1.0/) applies to the data made available in this article, unless otherwise stated in a credit line to the data. 
addition to the development of the typical white matter (WM) MS lesions, the disease is also characterized with neurodegenerative and vascular abnormalities [1]. Based on the Monro-Kellie doctrine, the sum of all volumes within the non-elastic cranium have to remain constant. Therefore, hemodynamic changes such as inflammationdriven hyperperfusion or atrophy-driven hypoperfusion will significantly influence the overall brain volume [2]. On the other hand, the continuous neurodegenerative process which leads to high global and central brain atrophy rates will contribute to substantial ex-vacuo enlargement of the ventricular space [3]. Thus, both processes can significantly influence intracranial pressure and thus affect the cerebrospinal fluid (CSF)-derived measures in MS patients. It is currently unknown whether the changes in cerebral fluid dynamics are driven by forces within the CSF space or due to atrophy-driven passive expansion.

A flow-sensitive, magnetic resonance imaging (MRI)based, phase contrast (PC) cine sequence can serve as a convenient and non-invasive method for quantitative assessment of anatomical and functional aspects of the CSF system $[4,5]$. In short, the PC images are generated by subtraction of two dataset which are acquired with bipolar (opposing) gradient sensitizations where the residual image phase shift allows velocity quantification. For best signal quality and to prevent aliasing artifacts, the encoded velocity $\left(\mathrm{V}_{\text {enc }}\right)$ should be calibrated approximately to the maximal anticipated flow velocity (in this case, maximal CSF velocity).

Several previous small and cross-sectional studies have investigated CSF flow dynamics in both MS patients and matched healthy controls ( $\mathrm{HCs}$ ) and have reported mixed results [6, 7]. Moreover, a recent MS study also suggested presence of underlying pulse wave encephalopathy which may share multiple pulsation pathophysiological mechanisms similar to the normal pressure hydrocephalus (NPH) [8]. Lastly, in a previous cross-sectional work of ours, we corroborated some presence of slightly altered CSF dynamics in MS patients when compared to HCs [9]. Furthermore, those findings were associated with more severe MRI outcomes including greater lesion volume and smaller central and WM volumes [9]. However, due to the limitations of cross-sectional design, all aforementioned studies were not equipped to provide insights regarding consequential relationships between changes in the CSF fluid dynamics and other MS-related MRI measures.

Until now, there are no reports which have longitudinally analyzed the changes of CSF measures in MS patients and HCs. Based on this background, we aimed to assess longitudinally the changes within AoS-derived variables including its size, average and peak CSF velocity and total CSF flow in both MS patients and age- and sexmatched HCs over a follow-up period of approximately 5 years.

\section{Materials and methods Study population}

The population used for this particular analysis was derived from a larger, longitudinal cardiovascular, environmental and genetic study in MS (CEG-MS) [10, 11]. The inclusion criteria for MS patients consisted of: (1) age between 18 and 75 years old at baseline; (2) availability of an anatomical MRI scan with an additional cine PC imaging protocol both at baseline and 5-year followup visits; (3) being MS as diagnosed per the 2010-revised McDonald criteria [12]. On the other hand, the exclusion criteria included (1) no clinically-defined relapse or use of intravenous corticosteroid within 30 days of the MRI examination, (2) pregnant or nursing mothers, and (3) presence of congenital malformations that affect the cerebrospinal fluid anatomy (ex. Chiari malformations, congenital hydrocephalus). The inclusion criteria for the HCs was: (1) age between 18 and 75 years old at baseline and (2) no presence of current nor history of past major neurological disorder.

The MS population underwent full clinical evaluation by experienced neurologist and was characterized by the Expanded Disability Status Scale (EDSS) scores [13]. Based on the clinical and radiological presentation, the patients were further classified in relapsing-remitting MS (RRMS) and progressive MS (PMS) subgroups.

\section{MRI acquisition and analysis}

Both MS patients and HCs underwent 3T MRI examination performed at baseline and 5-year follow-up on 3T GE Signa Excite HD 12 Twin Speed 8-channel scanner (General Electric, Milwaukee, WI, USA) and an 8-channel head and neck coil. There were no substantial hardware nor software changes over the follow-up period.

For CSF flow and velocity measurement, a 2D cine PC imaging with velocity-encoded pulse-gated sequence was acquired, perpendicularly to the AoS, with the following parameters: echo time (TE) of $7.9 \mathrm{~ms}$ and repetition time (TR) of $40 \mathrm{~ms}, 20 \mathrm{~cm} / \mathrm{s}$ velocity encoding, slice thickness of $2 \mathrm{~mm}$, flip angle (FLIP) of $20^{\circ}$, and pulse oximetergated procedure for obtaining 32 points of the cardiac cycle. In order to minimize potential flow variations due to different cranio-caudal levels or different head rotations, the acquisition protocol was standardized and the technician paid attention to avoid head tilting/rotation. The imaging plane was positioned at the level of the ampulla, perpendicularly to the AoS axis, visible on the midsagittal scout [14]. For brain volume measurements, MS patients underwent additional high-resolution 3D 
T1-weighted imaging with a spoiled gradient echo with inversion recovery preparation pulse with TE/inversion time (TI)/TR of $2.8 \mathrm{~ms} / 900 \mathrm{~ms} / 5.9 \mathrm{~ms}$, FLIP of $10^{\circ}$, field of view (FOV) of $25.6 \mathrm{~cm} \times 19.2 \mathrm{~cm}$, voxel size of $1 \times 1 \times 1 \mathrm{~mm}^{3}$ with no gaps.

For analysis of the PC cine imaging, freely available software Segment version 2.0 (MedViso, Lund, Sweden) was utilized [15]. The AoS-derived cross-sectional area expressed in $\mathrm{mm}^{2}$ (CSA) and velocity measures expressed in $\mathrm{cm} / \mathrm{s}$ for each pixel inside the CSA contours were derived for all 32 points of the cardiac cycle (Fig. 1). Using these measures were further computed: (1) average area of the AoS, (2) averaged velocity (considering all the pixel inside the segmented CSA; Vmean) for both systolic and diastolic peaks, (3) maximal velocity (pixel with highest velocity inside the segmented CSA; Vmax) for both systolic and diastolic peaks, (4) average CSF flow rate (Vmean*AoS CSA) and (5) systolic and diastolic flow volumes. On the other hand, to determine the rate of brain atrophy within the MS patients, the 3D T1-WI images were inpainted and then analyzed by Structural Image Evaluation using Normalisation of Atrophy (SIENA) and SIENAX multi-time point algorithm, where whole brain (WB) atrophy and ventricular CSF (vCSF) space expansion were calculated, respectively [16]. T1 and T2 lesions and their volume were segmented using validated semi-automated, contouring/thresholding procedure with Java Image Manipulation (JIM, Xinapse systems, Essex, UK) software. In short, SIENA software allows quantitative temporal brain atrophy measurement by utilizing two MRI images from different time points and producing a third "change" image. The images are coregistered to each other, scaled and skewed based on the skull input. Similarly, SIENAX produces intra-subject comparable volumes by using brain-and-skull based correction and scaling into standard MR space. SIENA and SIENAX demonstrate accuracy of $0.2 \%$ and $1 \%$ error in normalized brain volume analysis [17]. Furthermore, the thalamic atrophy was determined with FMRIB's Integrated Registration and Segmentation Tool (FIRST) software (FMRIB, Oxford, UK). The volumes of T2 lesion, $\mathrm{WB}, \mathrm{vCSF}$ and thalamus are expressed in milliliters $(\mathrm{ml})$ and in total and annualized longitudinal \% change, for baseline and longitudinal measures, respectively.

\section{Statistical analysis}

All statistical analyses were performed in SPSS 25.0 (IBM, Armonk, USA). The distribution of the data and their residuals was determined by Kolmogorov-Smirnov test for normality and by visual inspection of Q-Q plots. The demographic and clinical differences between MS and HCs were derived by parametric ( $\chi^{2}$ test, Student's t-test and analysis of covariance; ANCOVA) and nonparametric (Mann-Whitney U-test) comparisons tests,

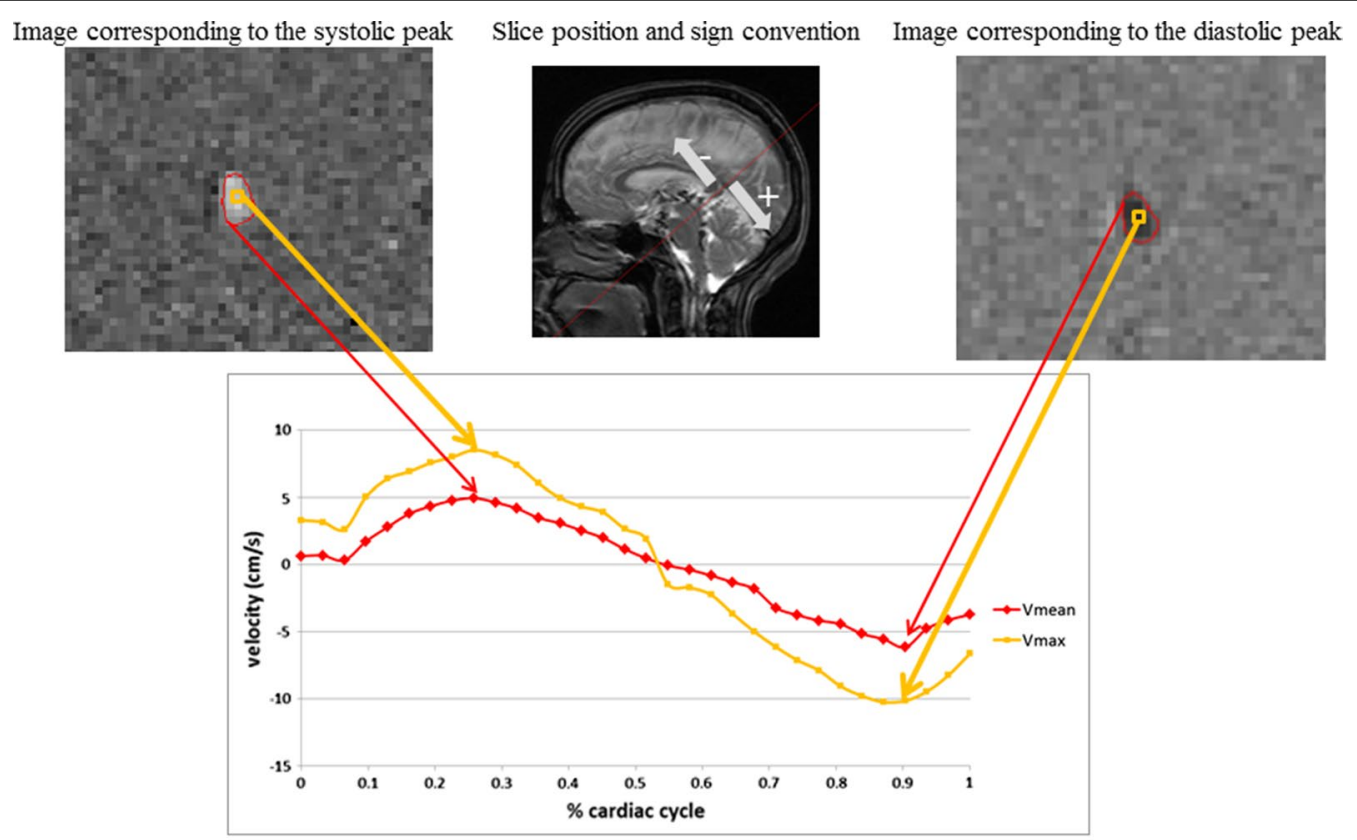

OVmean: average velocity inside the AoS ROI for each time point (mean of pixels)

a Vmax: maximum velocity inside the AoS ROI for each time point (one pixel)

Fig. 1 Cross-sectional area of Aqueduct of Sylvius and velocity measures. AoS Aqueduct of Sylvius, $R O /$ region of interest, $V$ velocity 
accordingly. The longitudinal change in AoS variables was determined by appropriate paired repeated measure tests. Lastly, repeated measure analysis within a general linear model (GLM) framework determined the significant effects and associations between the AoS variables and suspected study covariates (age, sex, disease phenotype, rate of global brain atrophy). Confirmatory regression analysis and partial Spearman's ranked correlations were performed. The aforementioned analyses were separately performed in the RRMS and PMS subtypes as well. The results were corrected for false discovery rate (FDR) utilizing Benjamini-Hochberg procedure. Accordingly, p-values and multiple comparison-corrected q-values $<0.05$ were considered statistically significant.

\section{Results}

\section{Demographic and clinical characteristics}

The demographic and clinical characteristics of the study population are shown in Table 1. Similarly, the specifics of the RRMS and PMS subgroups are also shown in Table 1. There were no differences between the MS patients and HCs in age (51.6 vs. 47.4 years old, t-test $\mathrm{p}=0.146$ ) nor in female/male ratio $\left(24 / 16\right.$ vs. $15 / 5, x^{2}$ test $\left.p=0.39\right)$. Similarly, there were no differences in the follow-up time between the MS patients and HCs $(5.6 \pm 0.6$ vs. $5.5 \pm 0.5$ years, $\mathrm{t}$-test $\mathrm{p}=0.858$ ). The MS population had an average disease duration of 16.2 years. Similarly, the median disability scores were EDSS 2.5 at both baseline and follow-up. The MS population consisted of 26 RRMS and 14 PMS patients. At baseline, 12 (30\%) MS patients were on interferon- $\beta, 12(30 \%)$ on glatiramer acetate, 7 $(17.5 \%)$ on natalizumab, and $9(22.5 \%)$ did not use any disease modifying treatment (DMT). During the 5-year follow-up, 29 (72.5\%) MS patients remained on the same DMT regiment, whereas $11(27.5 \%)$ patients have changed their medications. There were no differences in DMT regiment changes between the RRMS and PMS groups $\left(\chi^{2}\right.$ test $\left.\mathrm{p}=1.000\right)$ Lastly, the total MS population had an average of $13.8 \%$ increase in total T2 lesion volume, $2.6 \% \mathrm{WB}$ atrophy rate and $13.7 \%$ of vCSF space expansion rate.

As expected, the PMS group was older (56.7 vs. 48.8 years old, t-test $\mathrm{p}=0.002$ ), had longer disease duration (23.6 vs. 12.2 years, t-test $\mathrm{p}<0.001)$ and had greater EDSS scores at both baseline and follow-up (both Mann Whitney U-test $\mathrm{p}<0.001$ ) when compared to the RRMS group. Similarly to the total group, there were no differences in the follow-up time between the

Table 1 Demographic and clinical characteristics of the study population

\begin{tabular}{|c|c|c|c|c|c|c|}
\hline & $\begin{array}{l}\text { MS } \\
(n=40)\end{array}$ & RRMS (n = 26) & $\begin{array}{l}\text { PMS } \\
(n=14)\end{array}$ & HCs $(n=20)$ & $\begin{array}{l}\text { MS vs. HCs } \\
\text { p-value }\end{array}$ & $\begin{array}{l}\text { RRMS vs. PMS } \\
\text { p-value }\end{array}$ \\
\hline Female, n (\%) & $24(60)$ & $15(57.7)$ & $9(64.3)$ & $15(75)$ & 0.39 & 0.746 \\
\hline Age, mean $\pm S D$ & $51.6 \pm 9.1$ & $48.8 \pm 9.7$ & $56.7 \pm 4.9$ & $47.4 \pm 12.9$ & 0.146 & 0.002 \\
\hline Follow-up period, mean \pm SD & $5.6 \pm 0.6$ & $5.5 \pm 0.6$ & $5.7 \pm 0.6$ & $5.5 \pm 0.5$ & 0.858 & 0.423 \\
\hline Disease duration, mean $\pm \mathrm{SD}$ & $16.2 \pm 10.6$ & $12.2 \pm 9.0$ & $23.6 \pm 9.6$ & - & - & $<0.001$ \\
\hline EDSS at BL, median (IQR) & $2.5(1.5-4.75)$ & $1.5(1.5-2.5)$ & $5.5(3.4-6.5)$ & - & - & $<0.001$ \\
\hline EDSS at FU, median (IQR) & $2.5(1.5-6.0)$ & $2.0(1.5-3.0)$ & $6.0(4.0-6.5)$ & - & & $<0.001$ \\
\hline \multicolumn{7}{|l|}{ DMT use at baseline, n (\%) } \\
\hline Interferon- $\beta$ & $12(30)$ & $10(38.4)$ & $2(21.4)$ & - & - & 0.51 \\
\hline Glatiramer acetate & $12(30)$ & $6(23.1)$ & $6(42.9)$ & - & & \\
\hline Natalizumab & $7(17.5)$ & $4(15.4)$ & $3(21.4)$ & - & & \\
\hline No DMT & $9(22.5)$ & $6(23.1)$ & $3(21.4)$ & - & & \\
\hline $\mathrm{T} 2 \mathrm{LV}$ change, mean $\pm \mathrm{SD}$ & $13.8 \pm 34.4$ & $20.5 \pm 39.7$ & $0.9 \pm 14.6$ & - & & 0.095 \\
\hline Annualized T2 LV change, mean \pm SD & $2.5 \pm 6.6$ & $3.8 \pm 7.7$ & $0.2 \pm 2.6$ & - & - & 0.112 \\
\hline WBV loss, mean \pm SD & $-2.6 \pm 1.5$ & $-2.6 \pm 1.4$ & $-2.5 \pm 1.5$ & - & - & 0.831 \\
\hline Annualized WBV loss, mean \pm SD & $-0.43 \pm 0.22$ & $-0.48 \pm 0.27$ & $-0.46 \pm 0.27$ & - & - & 0.779 \\
\hline vCSF expansion, mean \pm SD & $13.7 \pm 10.5$ & $12.5 \pm 9.9$ & $15.8 \pm 11.6$ & - & - & 0.384 \\
\hline Annualized vCSF expansion, mean \pm SD & $2.29 \pm 1.8$ & $2.26 \pm 1.8$ & $2.89 \pm 2.1$ & - & - & 0.353 \\
\hline Thalamus atrophy, mean \pm SD & $-4.7 \% \pm 4.7$ & $-4.5 \% \pm 5.0$ & $-5.1 \% 4.3$ & - & - & 0.701 \\
\hline Annualized thalamus atrophy, mean \pm SD & $-0.85 \pm 0.88$ & $-0.81 \pm 0.9$ & $-0.92 \pm 0.8$ & - & - & 0.724 \\
\hline
\end{tabular}

The T2-LV, WBV, VCSF and thalamic volume changes are expressed in longitudinal \% change. The lesion and brain volumes are measured as milliliters ( $\mathrm{mL}$ )

MS multiple sclerosis, RRMS relapsing-remitting multiple sclerosis, PMS progressive multiple sclerosis, HCs healthy controls, EDSS Expanded Disability Status Scale, $D M T$ disease modifying treatment, $L V$ lesion volume, $W B V$ whole brain volume, $V C S F$ ventricular cerebrospinal fluid, $S D$ standard deviation, $I Q R$ interquartile range, $B L$ baseline, FU follow-up

$\mathrm{X}^{2}$ and Student's t-test were used. $\mathrm{p}$-value lower than 0.05 was considered statistically significant and shown in italics 
RRMS and PMS patients ( $5.5 \pm 0.6$ vs. $5.7 \pm 0.6$ years, t-test $\mathrm{p}=0.423)$ No differences in female/male ratio $\left(15 / 11\right.$ vs. $\left.9 / 5 \chi^{2} \mathrm{p}=0.746\right)$ nor in DMT use $\left(\chi^{2}\right.$ $\mathrm{p}=0.51)$ were noted. Lastly, there were no significant differences in total longitudinal T2 lesion volume $(0.9 \%$ vs. $20.5 \%$, t-test $\mathrm{p}=0.095)$ nor annualized $\%$ change $(0.2 \%$ vs. $3.8 \%$, t-test $\mathrm{p}=0.321)$, total nor annualized WB atrophy $(-2.5 \%$ vs. $-2.6 \%$, t-test $\mathrm{p}=0.831$ and $-0.46 \%$ vs. $-0.48 \%$, t-test $\mathrm{p}=0.779)$, total nor annualized vCSF expansion rate $(15.8 \%$ vs. $12.5 \%$, t-test $\mathrm{p}=0.384$ and $2.89 \%$ vs. $2.26 \%$, t-test $\mathrm{p}=0.353)$ and total nor annualized thalamic atrophy $(-4.7 \%$ vs. $5.1 \%$, $\mathrm{t}$-test $\mathrm{p}=0.701$ and $-0.92 \%$ vs. -0.8 , t-test $\mathrm{p}=0.724$ ).

\section{Phase contrast cine imaging variables}

Full description of the cross-sectional and longitudinal change of the AoS-derived variables at baseline and follow-up visits for both the MS and HCs populations are shown in Tables 2 and 3. Due to low phase signal quality of the scan, $5 \mathrm{HCs}$ and $5 \mathrm{MS}$ patients were excluded at the baseline visit, whereas $5 \mathrm{HCs}$ and 9 MS patients were excluded at the follow-up visit. In paired repeated measure analysis, the HCs demonstrated only significant increase in the maximal diastolic peak (from 6.31 to $7.61 \mathrm{~cm} / \mathrm{s}$, paired test $\mathrm{p}=0.035)$. On the other hand, the MS population demonstrated significant increase within the maximal diastolic peak (from 7.23 to $7.86 \mathrm{~cm} / \mathrm{s}$, paired test $\mathrm{p}=0.037)$, diastolic peak flow rate $(7.76 \mathrm{ml} /$

Table 2 Longitudinal analysis of phase contrast flow measures in both MS patients and HCs

\begin{tabular}{|c|c|c|c|c|c|c|}
\hline \multirow[t]{2}{*}{ AoS-derived measures } & \multicolumn{3}{|l|}{$\mathrm{HCs}(n=13)$} & \multicolumn{3}{|l|}{$M S(n=29)$} \\
\hline & Baseline & Follow-up & $\begin{array}{l}\text { BL to FU } \\
\text { p-value }\end{array}$ & Baseline & Follow-up & $\begin{array}{l}\text { BL to FU } \\
p \text {-value }\end{array}$ \\
\hline Vmean systolic peak (cm/s) & $5.10 \pm 2.31$ & $4.89 \pm 2.1$ & 0.579 & $5.46 \pm 2.16$ & $5.43 \pm 1.73$ & 0.939 \\
\hline Vmean diastolic peak (cm/s) & $3.75 \pm 2.28$ & $4.14 \pm 2.37$ & 0.204 & $4.22 \pm 1.32$ & $4.06 \pm 1.20$ & 0.415 \\
\hline Vmax systolic peak (cm/s) & $9.16 \pm 4.64$ & $9.88 \pm 5.03$ & 0.177 & $9.85 \pm 3.56$ & $9.54 \pm 2.97$ & 0.53 \\
\hline Vmax diastolic peak (cm/s) & $6.31 \pm 3.19$ & $7.61 \pm 4.35$ & 0.035 & $7.23 \pm 2.00$ & $7.86 \pm 2.76$ & 0.037 \\
\hline Average area $\left(\mathrm{mm}^{2}\right)$ & $2.62 \pm 1.20$ & $2.86 \pm 1.26$ & 0.19 & $3.12 \pm 1.07$ & $3.69 \pm 1.55$ & $0.001^{*}$ \\
\hline Flow rate systolic peak (ml/min) & $8.65 \pm 6.05$ & $8.78 \pm 5.12$ & 0.882 & $10.36 \pm 5.53$ & $12.16 \pm 7.30$ & 0.051 \\
\hline Flow rate diastolic peak (ml/min) & $6.57 \pm 5.59$ & $7.55 \pm 5.72$ & 0.207 & $7.76 \pm 3.51$ & $9.33 \pm 5.64$ & 0.023 \\
\hline Net vol caudal ( $\mu \mathrm{l} /$ beat) & $37.74 \pm 25.34$ & $33.29 \pm 19.41$ & 0.334 & $46.93 \pm 27.31$ & $50.49 \pm 34.4$ & 0.391 \\
\hline Net vol cranial ( $\mu \mathrm{l} /$ beat) & $37.89 \pm 36.75$ & $38.92 \pm 38.5$ & 0.885 & $41.13 \pm 23.56$ & $45.86 \pm 32.22$ & 0.208 \\
\hline
\end{tabular}

HCs healthy controls, MS multiple sclerosis, AoS Aqueduct of Sylvius, Vmean average velocity, Vmax maximal velocity, BL baseline, FU follow-up

All measures are shown as mean \pm standard deviation. Paired repeated measure analysis was utilized. $p$-values lower than 0.05 were considered statistically significant and shown in bold italics where trending values are shown in italics

* Statistically significant after false discovery rate correction

Table 3 Comparison of phase contrast flow measures between MS patients and HCs

\begin{tabular}{|c|c|c|c|c|c|c|}
\hline \multirow{2}{*}{ AoS-derived measures } & \multicolumn{3}{|l|}{ At baseline } & \multicolumn{3}{|l|}{ At follow-up } \\
\hline & $\mathrm{HCs}(n=15)$ & MS $(n=35)$ & $\begin{array}{l}\text { p-value } \\
\text { HCs vs MS }\end{array}$ & $\mathrm{HCs}(n=15)$ & $\operatorname{MS}(n=31)$ & $\begin{array}{l}\text { p-value } \\
\text { HCs vs. MS }\end{array}$ \\
\hline Vmean systolic peak (cm/s) & $5.07 \pm 2.29$ & $5.73 \pm 2.35$ & 0.611 & $4.70 \pm 1.91$ & $5.40 \pm 1.68$ & 0.222 \\
\hline Vmean diastolic peak $(\mathrm{cm} / \mathrm{s})$ & $-3.95 \pm 2.31$ & $-4.30 \pm 1.45$ & 0.639 & $-3.71 \pm 2.21$ & $-4.09 \pm 1.16$ & 0.408 \\
\hline Vmax systolic peak (cm/s) & $9.08 \pm 4.5$ & $9.82 \pm 3.48$ & 0.817 & $9.22 \pm 4.74$ & $9.53 \pm 2.87$ & 0.834 \\
\hline Vmax diastolic peak $(\mathrm{cm} / \mathrm{s})$ & $-6.5 \pm 3.16$ & $-7.32 \pm 2.13$ & 0.468 & $-6.76 \pm 4.1$ & $-7.82 \pm 2.68$ & 0.315 \\
\hline Average area $\left(\mathrm{mm}^{2}\right)$ & $2.53 \pm 1.14$ & $3.13 \pm 1.18$ & 0.325 & $2.57 \pm 1.27$ & $3.58 \pm 1.55$ & 0.045 \\
\hline Flow rate systolic peak (ml/min) & $8.35 \pm 5.85$ & $10.61 \pm 5.41$ & 0.508 & $7.71 \pm 5.02$ & $11.75 \pm 7.22$ & 0.072 \\
\hline Flow rate diastolic peak (ml/min) & $-6.60 \pm 5.38$ & $-7.92 \pm 3.58$ & 0.582 & $-6.32 \pm 5.49$ & $-9.10 \pm 5.52$ & 0.132 \\
\hline Net vol caudal ( $\mu$ l/beat) & $37.01 \pm 24.48$ & $47.42 \pm 26.23$ & 0.534 & $29.59 \pm 18.85$ & $49.25 \pm 33.63$ & 0.052 \\
\hline Net vol cranial ( $\mu \mathrm{l} /$ beat) & $-36.91 \pm 34.83$ & $-40.7 \pm 23.39$ & 0.895 & $-32.49 \pm 35.67$ & $-44.58 \pm 31.59$ & 0.302 \\
\hline
\end{tabular}

HCs healthy controls, MS multiple sclerosis, AoS Aqueduct of Sylvius, Vmean average velocity, Vmax maximal velocity

All measures are shown as mean \pm standard deviation. Analysis of covariance (ANCOVA) adjusted for age and sex was utilized. p-values lower than 0.05 were considered statistically significant and shown in bold italics where trending values are shown in italics 
$\min$ to $9.33 \mathrm{ml} / \mathrm{min}$, paired test $\mathrm{p}=0.023$ ) and in AoS CSA (from 3.12 to $3.69 \mathrm{~mm}^{2}$, paired test $\mathrm{p}=0.001$ ). Only the latter significant change survived FDR adjustment for multiple comparisons. In terms of between-group comparisons, at baseline, there were no statistical differences in any of the AoS-derived variables between MS patients and HCs. In line with the longitudinal changes, the only differentiator between MS patients and HCs was the greater AoS CSA measured at follow-up $\left(3.58 \mathrm{~mm}^{2}\right.$ vs. $2.57 \mathrm{~mm}^{2}$, age- and sex-adjusted ANCOVA, $\mathrm{p}=0.045$ ).

Moreover, the longitudinal change and differences of AoS-derived measures within and between the RRMS and PMS patients are shown in Additional file 1: Tables S1, S2. The increase of the AoS size over the follow-up was mainly driven by the increase seen within the PMS patients (from 3.24 to $3.96 \mathrm{~mm}^{2}$, paired test $\mathrm{p}=0.004$ ). This finding did survive FDR correction. Similarly the PMS group had greater annualized AoS CSA \% change when compared to the RRMS (6.3\% vs. $1.1 \%$, t-test $p=0.023)$. No significant differences in AoS-derived measures between the RRMS and PMS subgroups were noted.

\section{Longitudinal changes in Aqueduct of Sylvius measures and their associations with brain volumes}

In order to determine whether changes in AoS CSA were driven by increased CSF velocities or by local atrophy changes, we performed repeated measure GLM models. After adjusting for age and sex, the AoS CSA repeated measure factor had a trending interaction with the longitudinal vCSF expansion rate (Wilks' Lambda test $\mathrm{F}=4.124$, partial $\eta^{2}=0.171, \mathrm{p}=0.056$ ) but not with the baseline nor change in maximal velocity. In confirmatory analysis, the AoS CSA change was associated with vCSF expansion rate (age- and sex-adjusted Spearman's correlation $\mathrm{r}=0.496, \mathrm{p}=0.019$ ) and not with the baseline nor change in maximal velocity. After correcting for age and sex effects, the expansion rate of the vCSF space provided additional $23.8 \%$ of explained AoS CSA variance $\left(R^{2}=0.273, t=2.557\right.$, standardized $\beta=0.51$, and $\mathrm{p}=0.019$ ). Lastly, greater AoS CSA change was also associated with more thalamic atrophy in the PMS subgroup $(r=-0.663, p=0.036)$.

\section{Discussion}

This study demonstrates that MS patients exhibit significant enlargement in their AoS over mid-term follow-up period. These changes were greater when compared to the $\mathrm{HC}$ population and were not accompanied by significant changes in other flow-derived CSF measures. Furthermore, the dilatation of AoS was associated with the rate of global ventricular expansion which is commonly used as a proxy for central brain atrophy in MS patients.
These findings were corroborated by associations of thalamic atrophy and dilatation of the bordering AoS.

The complex and multifaceted disease-derived changes that occur within MS patients can influence the CSF dynamics. Based on the aforementioned Monro-Kellie doctrine, the recent findings of anatomical and functional cerebrovascular changes should also change the CSF parameters [18]. For example, the increased intracranial arterial volume (hyperperfusion) during acute inflammation would increase the overall brain volume and result with greater CSF velocities (greater amount of CSF would be propelled out of the cranium) [19]. On the other hand, MS patients also experience chronic narrowing of the major arterial vessels, decreased total amount of cerebral arterial blood flow, and cerebral hypoperfusion [20, 21]. Similar findings of decreased arterial blood flow were seen in a recent PC study which examined all three arterial, venous and CSF compartments [22]. The same study additionally showed lower CSF oscillations in the $19 \mathrm{MS}$ patients when compared to $21 \mathrm{HCs}$ [22]. Phase shifts during cardiac cycle seen in the arteriovenous compartment can directly influence the CSF pulsatility [23]. Such features can be seen in patients of idiopathic intracranial hypertension, where a small increase in arteriovenous pulsations resulted in greater CSF flow through the foramen magnum and increase in intracranial pressure [23]. Moreover, a decrease or loss in cerebrovascular reactivity function will result in the inability to adjust during changes in systemic arterial pressure and allow direct propagation of the pressure pulse into the CSF. This inability to dampen the pressure wave may contribute to greater CSF fluid velocities and oscillations $[24,25]$. MS patients may also display increased age- and atherosclerosis-driven arterial stiffening, which can further contribute towards extending the systolic pulse wave into the cerebral arterial vasculature [26]. In our MS sample, we were not able to demonstrate significant differences in CSF velocities when compared to the HCs nor significant changes after 5-year follow-up. Therefore, it does not support the notion of abnormal CSF dynamics within MS patients. Previous and similarly powered studies have shown largely discrepant results. A study which investigated 16 RRMS patients and $8 \mathrm{HCs}$ has demonstrated decreased net CSF flow [27]. Analogous findings of decreased net CSF flow were later demonstrated in a cohort of 67 MS patients and 9 clinically isolated syndrome patients when compared to $35 \mathrm{HCs}$ [9]. Contrarily, another study did not demonstrate differences in net CSF flow nor in CSF stroke volume between 21 RRMS patients and $20 \mathrm{HCs}$ [6]. Albeit it did not survive statistical multiple comparison correction, the increase in AoS CSA was accompanied by expected increase in CSF flow 
rate $(\mathrm{p}=0.023$ for diastolic flow rate peak and $\mathrm{p}=0.051$ for systolic flow rate peak).

On the other hand, we did demonstrate significant enlargement of the AoS, a finding which was particularly evident within the progressive MS patients. Moreover, the multiple regression analysis highlighted the relationship between longitudinal change in the size of AoS and global atrophy as measured by expansion of the total vCSF space and local changes as measured by thalamic atrophy. Furthermore, the statistical findings in this particular regression model did not include the baseline or the longitudinal change within the maximal velocity as a contributing factor that was associated with the change in AoS CSA. Therefore, these findings can potentially argue against internal fluid dynamics within the CSF space, but rather a passive dilatation of the AoS due to MS-driven neurodegenerative changes. Central brain atrophy measured as expansion of the third ventricle and/or as expansion of the lateral ventricles is one of the most reliable neurodegenerative MS biomarkers which have been strongly associated with clinical disability and disease progression [28]. The greater enlargement of the AoS within the PMS group is in line with the increasing MS literature that shows accelerated atrophy rate in these later stages of the disease [29]. Furthermore, recent findings have shown that the expansion of the ventricles may be due to atrophying periventricular lesions [30]. The chronic inflammatory processes that persist within these periventricular lesions would lead to absolute destruction of the brain tissue and its transformation into expanded CSF spaces [30]. An example of greater ventricular enlargement in an MS patient when compared to $\mathrm{HC}$ is shown in Additional file 2: Figure S1. These processes result in a non-uniform/non-symmetric expansion of the ventricles when compared to symmetrical expansion produced by high ventricular pressure and high CSF velocities. These findings are further corroborated by the fact that our PMS patients exhibited both numerically lower $\mathrm{T} 2$ lesion volume accrual and greater vCSF expansion rate over the 5-year follow-up period. Moreover, the thalamus, a highly interconnected brain hub, is particularly vulnerable structure affected by lesion-driven retrograde and/or anterograde (Wallerian) neurodegeneration [31]. Since the thalamus provides anatomical borders with the third ventricle and it is in the close proximity to the AoS, atrophic thalamus changes would significantly impact the AoS size.

Outside of the MS field, studies have utilized vascular and CSF dynamics in order to differentiate age-associated neuropathologies and the resulting ventricular expansion [32]. For example, the hydrodynamic abnormalities in amnestic mild cognitive impairment (aMCI) and the subsequent Alzheimer's disease (AD) may be initiated by changes in arterial flow changes first, whereas the hydrodynamic abnormalities in NPH may originate from changes within the CSF flow [33]. Our CSF hydrodynamic results also fall in line with measures in the aMCI and $\mathrm{AD}$ populations. When compared to NPH, MS patients and other neurodegenerative diseases display 4 to 5 times lower aqueductal stroke volume (30-40 $\mathrm{\mu l} /$ beat in MS/AD/aMCI vs. 160-170 $\mu \mathrm{l} /$ beat in NPH) [34]. Furthermore, the expansion in AoS CSA and ventricular volume seen NPH patients is associated with this aforementioned aqueductal stroke volume, whereas the AoS CSA change in the classical neurodegenerative diseases may be driven by extraventricular factors [34]. Lastly, the absolute stroke volume does increase with the normal processes of aging (33\% for every following year) and differs between sexes (20\% higher in males) [35]. Although we did statistically adjust for both demographic covariates, the small increase in systolic and diastolic flow rate seen in our MS population can be potentially explained by intensified aging brain interactions [35].

A limitation to our work is the relatively small number of MS patients and HCs. Further analysis between longitudinal changes in CSF measures and clinical disability accrual/phenotype progression should be considered. Also, future studies should include multiple PC sequences with different velocity encodings which would allow simultaneous examination of the changes within CSF fluid dynamics together with hemodynamic measures of the arterial and venous counterparts. Changes in AoS velocity, pressure gradients, and flow measures due to heart cycle changes, respiration or exercise should be monitored in future studies [36]. As such, a recent study demonstrated that respiratory-induced pressure gradients-the force driving the net CSF flow through the AoS-dominate over the cardiac component which is responsible for more frequent and pulsatile peak flow rates [37]. That being said, the PC-MRI measurements may mostly depict the short-term pressure fluctuations and poorly correlate with the overall intracranial pressure changes [38]. Lastly, attempts at MRI analysis of newly demonstrated cerebral glymphatic and lymphatic flow can contribute towards more comprehensive understanding of the brain fluid dynamics [39]. If brain fluid dynamics contribute towards the multifaceted pathophysiological MS mechanisms, either pharmacological or rehabilitative treatments may alleviate some the aforementioned abnormalities [40].

\section{Conclusions}

MS patients present with significant longitudinal AoS area dilatation, potentially due to regional atrophy changes and ex-vacuo expansion of the aqueduct. There were no significant changes in velocity and flow dynamics 
of the CSF measures. MS brain atrophy may be responsible for a significant part of the functional CSF changes previously seen in the MS population.

\section{Supplementary information}

Supplementary information accompanies this paper at https://doi. org/10.1186/s12987-020-0172-3.

Additional file 1: Table S1. Longitudinal analysis of phase contrast flow measures in RRMS and PMS subpopulations. Table S2. Comparison of phase contrast flow measures between RRMS and PMS patients.

Additional file 2: Figure S1. Ventricular enlargement example in MS patients when compared to HC. MS multiple sclerosis, HC healthy control, VCSF ventricular cerebrospinal fluid, A-MS patient demonstrating significant $80 \%$ enlargement of the ventricular CSF spaces. Note the ventricular expansion and the significant atrophy of the thalamus. B-healthy control demonstrating low rate of vCSF expansion over the same follow-up period.

\section{Abbreviations}

MS: multiple sclerosis; CNS: central nervous system; WM: white matter; CSF: cerebrospinal fluid; MRI: magnetic resonance imaging; PC: phase contrast; HCs: healthy controls; AoS: Aqueduct of Sylvius; EDSS: Expanded Disability Status Scale; RRMS: relapsing-remitting multiple sclerosis; PMS: progressive multiple sclerosis; TE: echo time; TR: repetition time; TI: inversion time; FLIP: flip angle; FOV: field of view; CSA: cross-sectional area; WBV: whole brain volume; VCSF: ventricular cerebrospinal fluid; ANCOVA: analysis of covariance; FDR: false discovery rate; GLM: general linear model; DMT: disease modifying treatment; IR-FSPGR: spoiled gradient echo with inversion recovery preparation; Vmean: average velocity; Vmax: maximal velocity; BL: baseline; FU: follow-up.

\section{Acknowledgements}

Not applicable.

\section{Authors' contributions}

DJ study concept and design, analysis and interpretation, manuscript drafting, critical revision of the manuscript for important intellectual content, study supervision. RZ, MML study concept and design; analysis and interpretation; critical revision of the manuscript for important intellectual content, study supervision. NB, MGD, BW analysis and interpretation; critical revision of the manuscript for important intellectual content. All authors read and approved the final manuscript.

\section{Funding}

Not applicable.

\section{Availability of the data and materials}

The datasets used and/or analyzed during the current study are available from the corresponding author on reasonable request.

\section{Ethics approval and consent to participate}

All study participants signed written consent form at both baseline and follow-up study visits. The study was approved by University at Buffalo, State University of New York Institutional Review Board (IRB).

\section{Consent for publication}

Not applicable.

\section{Competing interests}

Dejan Jakimovski, Niels Bergsland and Marcela Maria Lagana have nothing to disclose.Michael G. Dwyer received personal compensation from Novartis and EMD Serono, and financial support for research activities from Celgene, Novartis, Mapi Pharma, Keystone Heart, Protembis, and V-WAVE Medical.Bianca Weinstock- Guttman received honoraria as a speaker and as a consultant for Biogen Idec, Teva Pharmaceuticals, EMD Serono, Genzyme, Sanofi, Novartis and Acorda. Dr Weinstock-Guttman received research funds from Biogen Idec, Teva Pharmaceuticals, EMD Serono, Genzyme, Sanofi, Novartis, Acorda.Robert
Zivadinov received personal compensation from Celgene, EMD Serono, Genzyme-Sanofi, and Novartis for speaking and consultant fees. He received financial support for research activities from Genzyme-Sanofi, Novartis, Celgene, Mapi Pharma, Keystone Heart, Protembis and V-WAVE Medical.

\section{Author details}

${ }^{1}$ Buffalo Neuroimaging Analysis Center (BNAC), Department of Neurology, Jacobs School of Medicine and Biomedical Sciences, University at Buffalo, State University of New York, Buffalo, NY, USA. ${ }^{2}$ Center for Biomedical Imaging at Clinical Translational Science Institute, University at Buffalo, State University of New York, Buffalo, NY, USA. ${ }^{3}$ Jacobs Comprehensive MS Treatment and Research Center, Department of Neurology, Jacobs School of Medicine and Biomedical Sciences, University at Buffalo, State University of New York, Buffalo, NY, USA. ${ }^{4}$ MRI Laboratory, CADiTeR, IRCCS, Fondazione Don Carlo Gnocchi ONLUS, Via Alfonso Capecelatro 66, 20148 Milan, Italy.

Received: 9 November 2019 Accepted: 21 January 2020

Published online: 31 January 2020

\section{References}

1. Jakimovski D, Topolski M, Genovese AV, Weinstock-Guttman B, Zivadinov R. Vascular aspects of multiple sclerosis: emphasis on perfusion and cardiovascular comorbidities. Expert Rev Neurother. 2019;19(5):445-58.

2. Laganà MM, Shepherd SJ, Cecconi P, Beggs CB. Intracranial volumetric changes govern cerebrospinal fluid flow in the Aqueduct of Sylvius in healthy adults. Biomed Signal Process Control. 2017;36:84-92.

3. Zivadinov R, Jakimovski D, Gandhi S, Ahmed R, Dwyer MG, Horakova D, et al. Clinical relevance of brain atrophy assessment in multiple sclerosis. Implications for its use in a clinical routine. Expert Rev Neurother. 2016;16(7):777-93.

4. Battal B, Kocaoglu M, Bulakbasi N, Husmen G, Tuba Sanal H, Tayfun C. Cerebrospinal fluid flow imaging by using phase-contrast MR technique. Br J Radiol. 2011:84(1004):758-65.

5. Lagana MM, Chaudhary A, Balagurunathan D, Utriainen D, Kokeny P, Feng W, et al. Cerebrospinal fluid flow dynamics in multiple sclerosis patients through phase contrast magnetic resonance imaging. Curr Neurovasc Res. 2014;11(4):349-58.

6. Sundstrom P, Wahlin A, Ambarki K, Birgander R, Eklund A, Malm J. Venous and cerebrospinal fluid flow in multiple sclerosis: a case-control study. Ann Neurol. 2010;68(2):255-9.

7. Zivadinov R, Magnano C, Galeotti R, Schirda C, Menegatti E, WeinstockGuttman B, et al. Changes of cine cerebrospinal fluid dynamics in patients with multiple sclerosis treated with percutaneous transluminal angioplasty: a case-control study. J Vasc Interv Radiol. 2013;24(6):829-38.

8. Bateman GA, Lechner-Scott J, Lea RA. A comparison between the pathophysiology of multiple sclerosis and normal pressure hydrocephalus: is pulse wave encephalopathy a component of MS? Fluids Barriers CNS. 2016:13(1):18.

9. Magnano C, Schirda C, Weinstock-Guttman B, Wack DS, Lindzen E, Hojnacki D, et al. Cine cerebrospinal fluid imaging in multiple sclerosis. J Magn Reson Imaging. 2012;36(4):825-34.

10. Jakimovski D, Weinstock-Guttman B, Gandhi S, Guan Y, Hagemeier J, Ramasamy DP, et al. Dietary and lifestyle factors in multiple sclerosis progression: results from a 5-year longitudinal MRI study. J Neurol. 2019;266(4):866-75.

11. Jakimovski D, Gandhi S, Paunkoski I, Bergsland N, Hagemeier J, Ramasamy DP, et al. Hypertension and heart disease are associated with development of brain atrophy in multiple sclerosis: a 5-year longitudinal study. Eur J Neurol. 2019;26(1):87-e8.

12. Polman CH, Reingold SC, Banwell B, Clanet M, Cohen JA, Filippi M, et al. Diagnostic criteria for multiple sclerosis: 2010 revisions to the McDonald criteria. Ann Neurol. 2011;69(2):292-302.

13. Kurtzke JF. Rating neurologic impairment in multiple sclerosis: an expanded disability status scale (EDSS). Neurology. 1983;33(11):1444-52.

14. Lee JH, Lee HK, Kim JK, Kim HJ, Park JK, Choi CG. CSF flow quantification of the cerebral aqueduct in normal volunteers using phase contrast cine MR imaging. Korean J Radiol. 2004;5(2):81-6. 
15. Heiberg E, Sjogren J, Ugander M, Carlsson M, Engblom H, Arheden H. Design and validation of segment-freely available software for cardiovascular image analysis. BMC Med Imaging. 2010;10:1.

16. Dwyer MG, Bergsland N, Zivadinov R. Improved longitudinal gray and white matter atrophy assessment via application of a 4-dimensional hidden Markov random field model. Neuroimage. 2014;90:207-17.

17. Smith SM, Zhang Y, Jenkinson M, Chen J, Matthews PM, Federico A, et al. Accurate, robust, and automated longitudinal and cross-sectional brain change analysis. Neuroimage. 2002;17(1):479-89.

18. Belov P, Jakimovski D, Krawiecki J, Magnano C, Hagemeier J, Pelizzari L, et al. Lower arterial cross-sectional area of carotid and vertebral arteries and higher frequency of secondary neck vessels are associated with multiple sclerosis. AJNR Am J Neuroradiol. 2018;39(1):123-30.

19. Wuerfel J, Bellmann-Strobl J, Brunecker P, Aktas O, McFarland H, Villringer $A$, et al. Changes in cerebral perfusion precede plaque formation in multiple sclerosis: a longitudinal perfusion MRI study. Brain. 2004;127(Pt 1):111-9.

20. Pelizzari L, Jakimovski D, Lagana MM, Bergsland N, Hagemeier J, Baselli $\mathrm{G}$, et al. Five-year longitudinal study of neck vessel cross-sectional area in multiple sclerosis. AJNR Am J Neuroradiol. 2018;39(9):1703-9.

21. Jakimovski D, Benedict RH, Marr K, Gandhi S, Bergsland N, WeinstockGuttman B, et al. Lower total cerebral arterial flow contributes to cognitive performance in multiple sclerosis patients. Mult Scler. 2019. https:// doi.org/10.1177/1352458518819608.

22. ElSankari S, Baledent O, van Pesch V, Sindic C, de Broqueville Q, Duprez T. Concomitant analysis of arterial, venous, and CSF flows using phase-contrast MRI: a quantitative comparison between MS patients and healthy controls. J Cereb Blood Flow Metab. 2013;33(9):1314-21.

23. Capel C, Baroncini M, Gondry-Jouet C, Bouzerar R, Czosnyka M, Czosnyka Z, et al. Cerebrospinal fluid and cerebral blood flows in idiopathic intracranial hypertension. Acta Neurochir Suppl. 2018;126:237-41.

24. Marshall O, Lu H, Brisset JC, Xu F, Liu P, Herbert J, et al. Impaired cerebrovascular reactivity in multiple sclerosis. JAMA Neurol. 2014;71(10):1275-81.

25. Pelizzari L, Lagana MM, Baglio F, Bergsland N, Cecconi P, Viotti S, et al. Cerebrovascular reactivity and its correlation with age in patients with multiple sclerosis. Brain Imaging Behav. 2019. https://doi.org/10.1007/ s11682-019-00132-5.

26. Thomas O, Cain J, Nasralla M, Jackson A. Aortic pulsatility propagates intracranially and correlates with dilated perivascular spaces and small vessel compliance. J Stroke Cerebrovasc Dis. 2019;28(5):1252-60.

27. Zamboni P, Menegatti E, Weinstock-Guttman B, Schirda C, Cox JL, Malagoni AM, et al. The severity of chronic cerebrospinal venous insufficiency in patients with multiple sclerosis is related to altered cerebrospinal fluid dynamics. Funct Neurol. 2009;24(3):133-8.

28. Ghione E, Bergsland N, Dwyer MG, Hagemeier J, Jakimovski D, Paunkoski l, et al. Brain atrophy is associated with disability progression in patients with MS followed in a clinical routine. AJNR Am J Neuroradiol. 2018;39(12):2237-42.
29. Ghione E, Bergsland N, Dwyer MG, Hagemeier J, Jakimovski D, Paunkoski I, et al. Aging and Brain atrophy in multiple sclerosis. J Neuroimaging. 2019;29(4):527-35

30. Dwyer MG, Bergsland N, Ramasamy DP, Jakimovski D, Weinstock-Guttman B, Zivadinov R. Atrophied brain lesion volume: a new imaging biomarker in multiple sclerosis. J Neuroimaging. 2018;28(5):490-5.

31. Fuchs TA, Carolus K, Benedict RHB, Bergsland N, Ramasamy D, Jakimovski $D$, et al. Impact of focal white matter damage on localized subcortical gray matter atrophy in multiple sclerosis: a 5-year study. AJNR Am J Neuroradiol. 2018;39(8):1480-6.

32. Chiang WW, Takoudis CG, Lee SH, Weis-McNulty A, Glick R, Alperin N. Relationship between ventricular morphology and aqueductal cerebrospinal fluid flow in healthy and communicating hydrocephalus. Investig Radiol. 2009:44(4):192-9.

33. El Sankari S, Gondry-Jouet C, Fichten A, Godefroy O, Serot JM, Deramond $\mathrm{H}$, et al. Cerebrospinal fluid and blood flow in mild cognitive impairment and Alzheimer's disease: a differential diagnosis from idiopathic normal pressure hydrocephalus. Fluids Barriers CNS. 2011;8(1):12.

34. Ringstad G, Emblem KE, Geier O, Alperin N, Eide PK. Aqueductal stroke volume: comparisons with intracranial pressure scores in idiopathic normal pressure hydrocephalus. AJNR Am J Neuroradiol. 2015;36(9):1623-30.

35. Sartoretti T, Wyss M, Sartoretti E, Reischauer C, Hainc N, Graf N, et al. Sex and age dependencies of aqueductal cerebrospinal fluid dynamics parameters in healthy subjects. Front Aging Neurosci. 2019;11:199.

36. Spijkerman JM, Geurts LJ, Siero JCW, Hendrikse J, Luijten PR, Zwanenburg JJM. Phase contrast MRI measurements of net cerebrospinal fluid flow through the cerebral aqueduct are confounded by respiration. J Magn Reson Imaging. 2019;49(2):433-44.

37. Vinje V, Ringstad G, Lindstrom EK, Valnes LM, Rognes ME, Eide PK, et al. Respiratory influence on cerebrospinal fluid flow-a computational study based on long-term intracranial pressure measurements. Sci Rep. 2019;9(1):9732.

38. Ringstad G, Lindstrom EK, Vatnehol SAS, Mardal KA, Emblem KE, Eide PK. Non-invasive assessment of pulsatile intracranial pressure with phasecontrast magnetic resonance imaging. PLoS ONE. 2017;12(11):e0188896.

39. Louveau A, Da Mesquita S, Kipnis J. Lymphatics in neurological disorders: a neuro-lympho-vascular component of multiple sclerosis and Alzheimer's disease? Neuron. 2016;91(5):957-73.

40. Yang J, Shanahan KJ, Shriver LP, Luciano MG. Exercise-induced changes of cerebrospinal fluid vascular endothelial growth factor in adult chronic hydrocephalus patients. J Clin Neurosci. 2016;24:52-6.

\section{Publisher's Note}

Springer Nature remains neutral with regard to jurisdictional claims in published maps and institutional affiliations.

\footnotetext{
Ready to submit your research? Choose BMC and benefit from:

- fast, convenient online submission

- thorough peer review by experienced researchers in your field

- rapid publication on acceptance

- support for research data, including large and complex data types

- gold Open Access which fosters wider collaboration and increased citations

- maximum visibility for your research: over $100 \mathrm{M}$ website views per year
}

At BMC, research is always in progress.

Learn more biomedcentral.com/submissions 